\title{
Solar Wind Turbulent Heating by Interstellar Pickup Protons: 2-Component Model
}

\author{
Philip A. Isenberg ${ }^{\mathrm{a}}$, Sean Oughton ${ }^{\mathrm{b}}$, Charles W. Smith ${ }^{\mathrm{a}}$ \\ and William H. Matthaeus ${ }^{\mathrm{c}}$ \\ ${ }^{a}$ Inst. for Study of Earth, Oceans and Space, University of New Hampshire, Durham, NH 03824, USA \\ ${ }^{b}$ Department of Mathematics, University of Waikato, Hamilton 3240, New Zealand \\ ${ }^{c}$ Bartol Research Institute, University of Delaware, Newark, DE 19716 USA
}

\begin{abstract}
We apply a recently developed 2-component phenomenology to the turbulent heating of the core solar wind protons as seen at the Voyager 2 spacecraft. We find that this new description improves the model predictions of core temperature and correlation scale of the fluctuations, yielding excellent agreement with the Voyager measurements. However, the model fluctuation intensity substantially exceeds the Voyager measurements in the outer heliosphere, indicating that this picture needs further refinement.
\end{abstract}

Keywords: Interstellar pickup ions, solar wind, turbulence

PACS: 96.60.Vg; 94.05.Lk.

\section{INTRODUCTION}

The ionization and pickup of inflowing interstellar hydrogen has large-scale effects on the supersonic solar wind [1-3]. The pickup process adds energy and momentum to the wind, resulting in heating and deceleration, which have been observed by the Voyager 2 plasma instrument. The observed deceleration [4,5] is consistent with theoretical predictions for the inflowing hydrogen density of $N_{H} \sim 0.1 \mathrm{~cm}^{-3}$ at the termination shock [6,7]. However, the plasma instrument on Voyager 2 is not able to directly detect the pickup protons, which remain in an energetic halo about the much cooler proton core. Thus, the measured temperatures at Voyager do not represent the total proton temperature, which should increase linearly with heliocentric radius. At the same time, the observed core temperatures do exhibit substantial heating beyond 20 AU [8,9], where the only plausible energy source would be a process which taps into the large pool of thermal energy in the pickup protons.

Models of this heating [10-16] suggest that the energy transfer to the thermal core protons is mediated by a turbulent cascade. The turbulence can be driven by shear flows in the inner heliosphere, but further from the Sun the energy comes from the waves generated when the pickup protons are scattered toward isotropy. At the very large scales governing the plasma in the outer heliosphere, it is particularly appropriate to represent the turbulence with a phenomenological description. Such models have applied simple parameterizations of the turbulent evolution in the spherically expanding solar wind, given the energy sources and a cascade of energy at the Kolmogorov rate to a dissipation which heats the core protons. These models are continually being refined and applied to more detailed comparisons with the data, 
yielding valuable information and insights into the processes of turbulent transport, wave instabilities, and pitch-angle scattering.

In the simplest version of these models, the nonlinear first-order steady-state equations follow two parameters: the turbulent intensity at the energy-containing scales, $Z^{2}=<\delta v^{2}>+<\delta b^{2} / 4 \pi \rho>$, and the correlation length of the turbulent fluctuations, $\lambda$. The solar wind core temperature is then given by a subsidiary equation which equates the heating with the turbulent dissipation at the cascade rate, and includes the adiabatic effects of spherical expansion.

Previous modeling efforts have found that the pickup proton fluctuation source depends sensitively on the resonant interaction with ambient quasi-parallel wave spectra $[13,14]$. Wave generation is due to the unstable, newly-ionized portion of the pickup distribution, and beyond $20 \mathrm{AU}$ this ionization rate is much smaller than the nonlinear cascade rate which distributes the unstable wave power across the spectrum. In what we term the "dominant turbulence" approximation, we have assumed that the turbulence maintains a power-law spectral shape in the inertial range with equal intensities in all four quasi-parallel transverse wave modes. The interaction with this wave spectrum yields a particular shape of the pickup shell in velocity space. The remaining energy determines the magnitude of the fluctuation source driving the turbulence, and this magnitude is seen to match well with observations of solar wind core temperature and fluctuation intensity.

This basic phenomenological model has been extended in several ways. The effect of deceleration through momentum loading by the pickup protons has been incorporated [17]. A non-zero cross-helicity of the turbulence has been added to the description $[16,18,19]$, although this quantity is not likely to be important for pickup in the azimuthal field of the outer heliosphere and will not be considered here. Further, the effect of replacing the Kolmogorov phenomenology with an Iroshnikov-Kraichnan cascade is being investigated [20]. The most detailed tests have come from a point-bypoint comparison of solar-rotation-averaged Voyager measurements with the predictions of the model, using observed solar wind conditions at $1 \mathrm{AU}$ for the model's inner boundary $[15,17]$.

Figure 1 shows a recent comparison with Voyager measurements which includes the solar wind deceleration due to pickup [17]. Here, the circles are the three-solarrotation averages of the observed core temperature at Voyager and the line shows the results of the turbulent heating model. This model still assumes a steady-state flow,

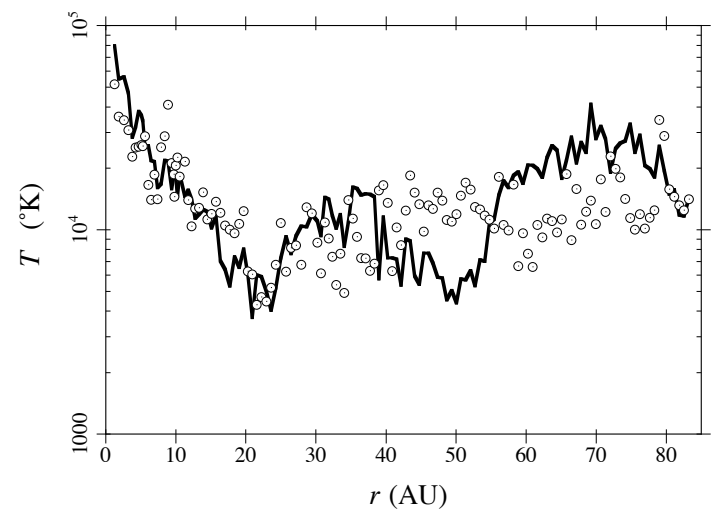

FIGURE 1. Time-variable results for core proton temperature from a one-component model, compared to three-solar-rotation averaged Voyager measurements. After [17]. but takes the 1 AU inner boundary condition for each model point from the observed solar wind conditions during the appropriately time-shifted period from the NSSDC Omnitape. The model predictions agree very well with the Voyager measurements when $r<40$ AU. The large discrepancy between 40 $55 \mathrm{AU}$ is understood as the effect of strong latitudinal structure in the solar wind during solar minimum. During this time, the wind conditions seen at Earth and input to the model do not correspond to the $1 \mathrm{AU}$ conditions of the high-latitude wind reaching Voyager. 
However, when Voyager has reached 60 AU, solar maximum has returned and the latitudinal gradients of the wind should be small. We see that the model temperatures in this region are substantially higher than observed.

In this paper, we apply another physically-motivated extension of the phenomenological model to see if this comparison can be improved.

\section{2-COMPONENT TURBULENCE MODEL}

One perceived shortcoming of the models described above is the limitation of the turbulence to a single fluctuation field, governed by behavior more appropriate to isotropic hydrodynamic turbulence or quasi-2D MHD. In the solar wind, the strong, large-scale magnetic field causes the turbulence to be anisotropic, and observations of solar wind turbulence have long indicated that there are at least two distinct types of fluctuations which probably evolve differently [21-23]. The "quasi-2D" fluctuations, which cascade primarily to larger $k_{\perp}$, have nonlinear interactions not strongly affected by propagation along the large-scale field. These fluctuations are expected to be governed by Kolmogorov-like phenomenology, as has been assumed above. The other type of fluctuations can be characterized as "wave-like", and their nonlinear interactions are influenced by propagation effects. A "2-component" phenomenological model has been introduced to treat this distinction [24,25].

Such a model may be especially important for describing the pickup-proton-driven turbulence in the outer heliosphere. The instabilities triggered by the newly-ionized protons are known to generate Alfvén-ion cyclotron waves with wavevectors concentrated along the large-scale magnetic field [26,27]. Thus, the fluctuation energy added to the turbulence from this source is likely to be wave-like and not well treated by the quasi-2D phenomenology of the one-component models.

In this new model, the two components of the turbulence are designated by $Z^{2}$ and $W^{2}$, to correspond to the quasi-2D and wave-like fields, respectively. Here, each fluctuation field evolves through its own nonlinear self-interactions, as well as through cascade and exchange interactions with the other field. The additional fluctuation field and implied anisotropy also require additional model variables to designate the energy-containing scales of each field in the parallel and perpendicular directions.

For zero cross-helicity in a spherically expanding wind of speed $U(r)$, with $U^{2}>>$ $V_{A}{ }^{2}>Z^{2}$ and $W^{2}$, the steady-state 2-component equations are

$$
\begin{aligned}
& \frac{d Z^{2}}{d r}=-A_{Z} \frac{Z^{2}}{r}-\frac{\alpha}{U}\left(\frac{Z^{3}}{\ell}+\frac{W Z^{2}}{\ell} \frac{2}{1+Z / W}\right)+\frac{\alpha}{U} X \\
& \frac{d W^{2}}{d r}=-A_{W} \frac{W^{2}}{r}-\frac{\tilde{\alpha}}{U}\left(\frac{W^{2} Z}{\lambda} \frac{2}{1+\lambda / \ell}+\frac{2 W^{4}}{\lambda^{2}} \frac{\lambda}{V_{A}}\right)-\frac{\alpha}{U} X+\frac{Q}{U}
\end{aligned}
$$




$$
\begin{aligned}
& \frac{d \ell}{d r}=C_{Z} \frac{\ell}{r}+\frac{\beta}{U}\left(Z+\frac{2 W}{1+Z / W}\right)-\frac{\beta}{U} \frac{\ell}{Z^{2}} X \\
& \frac{d \lambda}{d r}=C_{W} \frac{\lambda}{r}+\frac{\tilde{\beta}}{U}\left(\frac{2 Z}{1+\lambda / \ell}+\frac{2 W^{2}}{\lambda} \frac{\lambda_{\|}}{V_{A}}\right)+\frac{\tilde{\beta}}{U}\left(\frac{\alpha}{\tilde{\alpha}} X \frac{\lambda}{W^{2}}\right) \\
& \frac{d \lambda_{\|}}{d r}=C_{W} \frac{\lambda_{\|}}{r}+\frac{2 \beta_{\|}}{U} \frac{W^{2} \lambda_{\|}^{2}}{V_{A} \lambda^{2}}-\left(\lambda_{\|}-\lambda_{r e s}\right) \frac{Q}{U W^{2}}
\end{aligned}
$$

where $\ell$ and $\lambda$ are the perpendicular correlation lengths for $Z$ and $W$, respectively, $\lambda_{\|}$is the parallel correlation scale for $W$, and the parallel scale for $Z$ has been set equal to $V_{A} \ell / Z$ [24]. The various $A$ 's and $C$ 's are physical constants parameterizing the combined effects of shear driving, spherical expansion, and deceleration by pickup. The various alphas and betas are order-one constants set by considerations of local turbulence theory, $Q$ is the rate of energy addition from the pickup protons, and $X$ is the rate of energy exchange between the $W$ and $Z$ fields given by

$$
X=2 W Z\left(W^{2}+Z^{2}\right)\left(\frac{1}{\lambda^{2}}-\frac{1}{\ell^{2}}\right) \frac{\lambda_{\|}}{V_{A}} .
$$

The pickup proton driving adds energy directly only to the $W$ field, at the scale $\lambda_{\text {res }}$ $=2 \pi V_{s w} / \Omega$, where $\Omega$ is the proton gyrofrequency. The solar wind core temperature is then given by the dissipation of each turbulent field, along with the adiabatic changes from expansion and deceleration

$$
\frac{d T}{d r}=-\frac{4 T}{3 r}-\frac{2 T}{3 U} \frac{d U}{d r}+\frac{m}{3 k_{B} U}\left[\alpha\left(\frac{Z^{3}}{\ell}+\frac{W Z^{2}}{\ell} \frac{2}{1+Z / W}\right)+\tilde{\alpha} \frac{W^{2}}{\lambda}\left(\frac{2 Z}{1+\lambda / \ell}+\frac{2 W^{2}}{\lambda} \frac{\lambda_{\|}}{V_{A}}\right)\right]
$$

\section{MODEL RESULTS}

To restrict the parameter space to a manageable size, we set $\alpha=\tilde{\alpha}, \beta=\beta=\beta_{\|}$, and $\alpha=2 \beta$. Solutions of this system with constant parameter values at the inner radial boundary have been presented in [25]. We obtain similar behavior, but we do not have the space to display the figures here. We find that $Z^{2}$ declines steadily with radius while the other variables increase. In particular, the $W^{2}$ field comes to dominate the total turbulent energy beyond $\sim 30$ AU. When we vary $\alpha$, maintaining the above relations between the alphas and betas, we see that the core temperature increases for smaller $\alpha$. We also consider other observable quantities, a weighted correlation length $L=\left(Z^{2} \ell+W^{2} \lambda\right) /\left(Z^{2}+W^{2}\right)$ and the fluctuation intensity of the magnetic field component normal to the ecliptic $<\delta B_{N}^{2}>=4 \pi \rho\left(Z^{2}+W^{2}\right) / 3$. Increasing $\alpha$ increases 

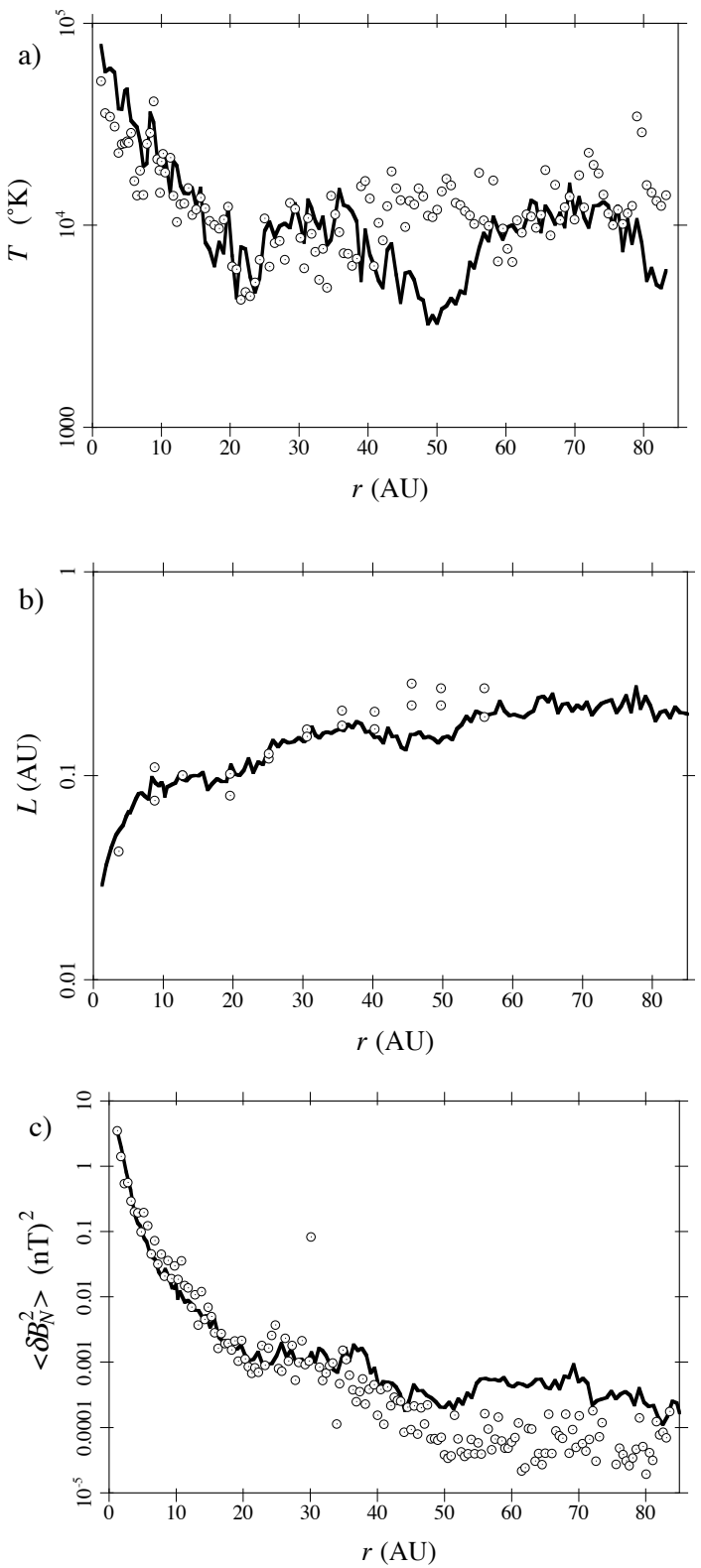

FIGURE 2. Time-variable results from the 2component model, compared to three-solarrotation averaged Voyager measurements. a) Core temperature. b) Weighted correlation length. c) Normal magnetic fluctuation intensity. the correlation length. This change also decreases the total fluctuation intensity somewhat, but only inside 30 AU. Further out, changing $\alpha$ seems not to affect $<\delta B_{N}{ }^{2}>$ at all in this model.

Next, we apply the same variable inner boundary procedure at 1 AU that was used to produce Figure 1. The observable solar wind quantities at Earth are averaged over a time-shifted period equivalent to each averaged Voyager measurement to set the boundary conditions. Additional assumptions at 1 AU take $\ell=\lambda=0.027 \mathrm{AU}, \lambda_{\|}=0.005$ $\mathrm{AU}$, and $W^{2} / Z^{2}=0.1$ there. Figure 2 shows the resulting core temperature, weighted correlation length, and fluctuation intensity compared to the three-solar-rotation averages of Voyager measurements (shown as circles) for $\alpha=$ 0.25 . While the analysis to obtain the correlation lengths could not proceed beyond $r=56 \mathrm{AU}$ due to frequent data gaps, the temperature and fluctuation intensity observations include the entire region inside the termination shock, which Voyager 2 crossed at $r=84$ AU. We see that the predicted temperatures and correlation lengths are in excellent agreement with the observations, except for the solar minimum period as explained above. We can even discern the beginning of the latest solar minimum for $r>80$ AU, just before Voyager leaves the supersonic solar wind.

However, Figure 2c shows that the predicted fluctuation intensity remains far higher than that observed in the solar wind. We note that this quantity is not affected by changes in $\alpha$, so a simple adjustment of parameters is not likely to improve this result. One possibility to keep in mind is that the propagating wave-like fluctuations may be concentrated in the parallel direction, giving $\mathbf{k} \bullet \mathbf{r} \sim 0$ in the highly azimuthal field of the outer heliosphere. In this case, it may be difficult to accurately measure the dominant $W$ component with the single point observations at Voyager. More probably, this discrepancy may highlight the need for improvements in this still preliminary model of turbulent evolution. 


\section{SUMMARY}

We report on our continued investigations into the turbulent heating of the distant solar wind driven by the pickup of interstellar hydrogen. In this paper, we apply a recently developed 2-component phenomenology to model the steady-state radial evolution of solar wind parcels, comparing the results with the measurements at Voyager 2. We find excellent agreement with the observed core proton temperature and with the correlation scale. However, the predicted fluctuation intensity is much higher than that observed beyond $r=30 \mathrm{AU}$. We conclude that this driven turbulent dissipation remains a viable mechanism to explain the observed core heating, but that further refinements of the phenomenological model appear to be necessary.

\section{ACKNOWLEDGMENTS}

We are grateful to Mel Goldstein and Gary Zank for valuable conversations. This work was supported in part by NSF grant ATM 0635863, NASA grants NNX07AH75G and NNX08A147G, and New Zealand Marsden Fund grant 02-UOW050 MIS.

\section{REFERENCES}

1. T. E. Holzer, J. Geophys. Res. 82, 23 (1977).

2. P. A. Isenberg, J. Geophys. Res. 91, 9965 (1986).

3. G. P. Zank, Space Sci. Rev. 89, 413 (1999).

4. C. Wang, J. D. Richardson, and J. T. Gosling, Geophys. Res. Lett. 27, 2429 (2000).

5. C. Wang, and J. D. Richardson, J. Geophys. Res. 108, 1058 (2003).

6. G. Gloeckler, L. A. Fisk, and J. Geiss, Nature 386, 374 (1997).

7. M. Bzowski, et al., Space Sci. Rev. 143, 177 (2009).

8. J. D. Richardson, et al., Geophys. Res. Lett. 22, 325 (1995).

9. J. D. Richardson, and C. W. Smith, Geophys. Res. Lett. 30, 10.1029/2002GL016551 (2003).

10. G. P. Zank, W. H. Matthaeus, and C. W. Smith, J. Geophys. Res. 101, 17,093 (1996).

11. W. H. Matthaeus, G. P. Zank, C. W. Smith, and S. Oughton, Phys. Rev. Lett. 82, 3444 (1999).

12. C. W. Smith, et al., J. Geophys. Res. 106, 8253 (2001).

13. P. A. Isenberg, C. W. Smith, and W. H. Matthaeus, Astrophys. J. 592, 564 (2003).

14. P. A. Isenberg, Astrophys. J. 623, 502 (2005).

15. C. W. Smith, et al., Astrophys. J. 638, 508 (2006).

16. B. Breech, et al., J. Geophys. Res. 113, A08105 (2008).

17. P. A. Isenberg, et al., Astrophys. J. submitted (2010).

18. W. H. Matthaeus, et al., Geophys. Res. Lett. 31, L12803 (2004).

19. B. Breech, et al., Geophys. Res. Lett. 32, L06103 (2005).

20. C. S. Ng, et al., J. Geophys. Res. 115, A02101 (2010); see also this volume.

21. W. H. Matthaeus, M. L. Goldstein, and D. A. Roberts, J. Geophys. Res. 95, 20,673 (1990).

22. J. W. Bieber, W. Wanner, and W. H. Matthaeus, J. Geophys. Res. 101, 2511 (1996).

23. S. Dasso, L. J. Milano, W. H. Matthaeus, and C. W. Smith, Astrophys. J. 635, L181 (2005).

24. S. Oughton, P. Dmitruk, and W. H. Matthaeus, Phys. Plasmas 13, 042306 (2006).

25. S. Oughton, W. H. Matthaeus, C. W. Smith, and B. Breech, in Solar Wind 12 M. Maksimovic, et al., Eds. Amer. Instit. Phys, Melville, NY, p. 210, (2010).

26. C. S. Wu, and R. C. Davidson, J. Geophys. Res. 77, 5399 (1972).

27. M. A. Lee, and W.-H. Ip, J. Geophys. Res. 92, 11,041 (1987). 